\title{
APPROACH TO THE DESIGN OF ROBUST NETWORKED CONTROL SYSTEMS
}

\author{
Michą MORAWSKI，ANTONi M. ZAJACZKOWSKI \\ Institute of Information Technology \\ Technical University of Łódź, Stefanowskiego 18/22, 90-934 Łódź, Poland \\ e-mail: \{morawski, zajaczko\}@zsk.p.lodz.pl
}

\begin{abstract}
The paper describes the application of the traffic engineering framework together with application layer procedures as mechanisms for the reduction of network latency lags. These mechanisms allow using standard and inexpensive hardware and software technologies typically applied for office networking as a means of realising networked control systems (NCSs) with high dynamic control plants, where a high dynamic control plant is the one that requires the sampling period several times shorter than communication lags induced by a network. The general discussion is illustrated by experimental results obtained in a laboratory NCS with the magnetic levitation system (MLS), which is an example of a structurally unstable plant of high dynamics.
\end{abstract}

Keywords: networked control systems, traffic engineering, industrial communication.

\section{Introduction}

In the last decade we have been observing a growing interest in design and implementation methods for NCSs (Zurawski, 2009). Typically, in an NCS, a plant is controlled or monitored by a remote computer system connected with the plant via a communication channel performed by a computer network (Hristu-Varsekelis and Levine, 2005). This architecture of the control system differs significantly from the classical one, where all system components are attached directly to the control plant and exchange data using some wiring system. NCSs gain popularity due to the fact that they can be implemented more cost effectively and can provide extended functionality compared with classical control systems. In particular, NCSs allow designing and implementing systems with gradual degradation, which is necessary in the case of many industrial control plants.

The application of computer networks for data exchange in automated production systems is not a new idea and during the last decades several industrial standards of computer networks have been developed. Industrial networks are typically based on asynchronous links (fieldbus) or technologies developed for specific areas. In this last category we have Profibus (Mitchell, 2004), ProfiNET (Nutzerorganisation, 2006), CAN (Pfeifer et al., 2003), ARINC (ARINC, 1990), WorldFIP (http: / / www. worldfip.org, n.d.), and many others.

The most important feature of these industrial networks is that they guarantee bounded transmission delays. However, NCSs based on industrial standards suffer from some disadvantages, like high installation and maintenance costs, excessive weight of physical links, and difficulties with scalability and redundancy. One of the solutions enabling partial avoidance of these problems is the application of standard, inexpensive, easy to purchase and replace devices typically used for office networking. They employ the Ethernet which currently dominates general networking and may become an industrial standard in the near future (Nutzerorganisation, 2006; Decotignie, 2005).

It is well known that incorrect operation of a control system due to some hardware failure can be dangerous or even catastrophic. Therefore, in order to increase the reliability of computer controlled systems, redundancy realised by the duplication or multiplication of sensors, actuators and selected processing units is applied, especially in the case of systems responsible for controlling critical processes.

In network-based control systems the situation is additionally complicated by the possibility of network failures and stochastically time-varying transmission delays introduced into feedback loops by a network. Hence, the resiliency of communication is crucial for satisfactory operation of these systems. It is obvious that the multiplication of communication links is not sufficient to assure 
a proper operation of NCSs and should be complemented by suitable traffic engineering and some procedures at the application level. While there is a need to keep network introduced delays below a certain bound, traffic engineering is uncomplicated when using point-to-point links, more complicated when using shared media, and quite complex in the case of wireless links prone to collisions, noise and interferences.

In the paper only an application and network level resiliency are considered. The physical (i.e., modulation and coding) and data link (frames and MAC procedures) layers 1 are defined by the appropriate standards and therefore cannot be easily altered. The significant influence of the link layer on latencies cannot be exploited in practise due to limitations of available hardware, firmware, and drivers. In particular, the implementation of the advanced 802.11e substandards like burst acknowledgements, no acknowledgements and $\mathrm{HCF}^{2}$ is extremely seldom, and software support for wider deployed $\mathrm{WMM}^{3}$ extensions is manufacturer dependent.

From the above discussion it follows that, in order to enhance the robustness of an NCS against network induced delays, an appropriate method of controller design should be supplemented by some methods of traffic engineering. The efficiency of this approach has been verified experimentally in our NCS with the MLS, which is an example of a structurally unstable and relatively fast control plant. These properties of the control plant cause that the sampling period used by a discrete-time controller must be several or even tens of times shorter than the delays introduced by the computer network into the feedback loop.

A result of this requirement was our choice of the method used for controller design. Namely, we applied a recently developed approach based on state prediction of the discrete-time linear model of the control plant (Liu et al., 2005; 2007; Yang et al., 2005). This approach belongs to the class of methods exploiting a deterministic mathematical model of the plant. In this class we also have the well-known model-based approach developed by Montestruque and Antsaklis (2003; 2005). From our point of view the main difference between these two methods is that the first one is well suited for problems requiring efficient compensation of network introduced delays, while the second one is oriented towards increasing the sampling period and, as a consequence, the reduction in the bandwidth required for stable operation of an NCS. In our application the control plant is unstable and of high dynamics, and thus we have to use a rather short sampling period to obtain satisfactory behaviour of the network ba-

\footnotetext{
${ }^{1}$ This assumption includes the case of real-time versions of Ethernet.

${ }^{2}$ HCF: Hybrid Coordination Function, a specification that distinguishes normal and prioritised (without contention) access to the medium.

${ }^{3}$ WMM: Wireless MultiMedia Extensions, a specification that allows distinguishing classes of service in wireless transmission.
}

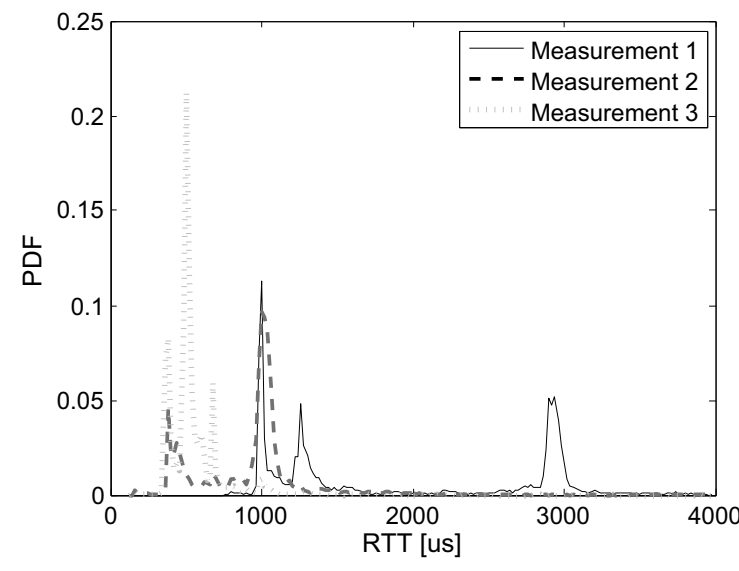

Fig. 1. Probability density functions of the RTT obtained in the laboratory network.

sed closed-loop system with variable delays several times longer than the sampling period. Hence, in our NCS, we implemented a remote controller designed using the first of the two aforementioned methods. The predictive controller implemented in the application layer was complemented by traffic engineering mechanisms developed recently by Morawski (2005; 2006b; 2007a; 2007b) and implemented in the network layer. It is worth adding that our experimental NCS was assembled from standard inexpensive network components typically used for office networking.

Summing up, the main goal of our research reported here is to demonstrate that the predictive control approach combined with appropriate traffic engineering mechanisms allows designing a robust NCS with the control plant of high dynamics and that such an NCS can be realised using general purpose network devices.

The organisation of the paper is as follows: in Section 2, delays introduced by the computer network are discussed, Section 3 describes other components of the link cost, in Section 4 the compensation of network introduced delays in the application layer is presented together with experimental results illustrating the properties of the laboratory NCS with the MLS. Section 5 presents an approach to network layer compensation of latencies, and Section 6 contains conclusions.

\section{Network introduced delays}

In classical networks (or high performance networks), the quality of the link (i.e., the cost of the link) is described by its existence only or by its physical bandwidth (discussed in the next section) or delay. Forwarding packets based on the principle of minimum delay is proven (Gallager, 1977) to be optimal. Unfortunately, the information about delays is always outdated and former approaches exploiting this rule have failed (Khanna and Zinky, 1989). 


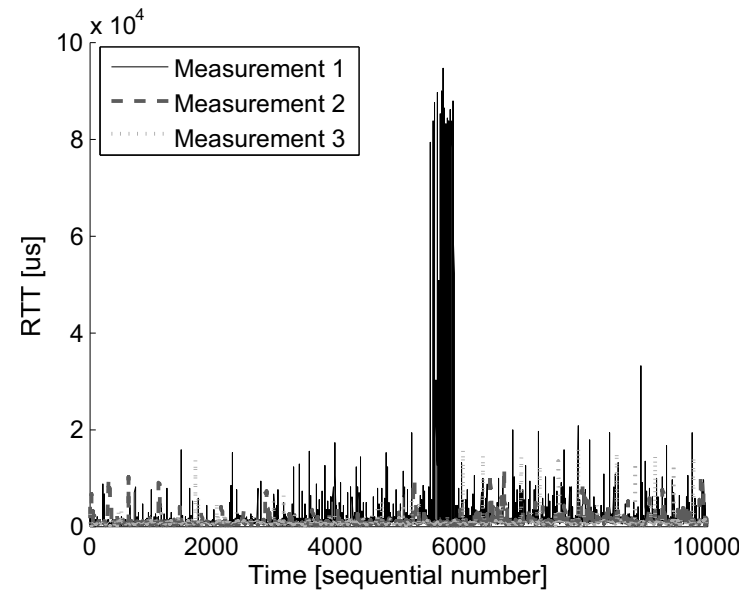

Fig. 2. RTTs time series in the laboratory network.

In many publications (e.g., Stallings, 2002), inevitable data transmission latencies introduced by computer networks are modelled statistically by some well-known distributions like exponential, Poisson, Pareto, gamma, Erlang, etc. Such models can be accepted in the case of the Internet network, where the main source of delays are queues in intermediate nodes. In the real-time traffic generated by NCSs, especially those using wireless channels, the significant influence of the media access procedure and Layer 2 retransmissions can be observed, and experimentally obtained latency distributions cannot be approximated by aforementioned models. This observation was confirmed by a number of experiments performed in our laboratory (Morawski, 2006a).

Figure 1 presents probability density functions (PDFs) of round trip times (RTTs) obtained by measurements in the network with Access Point 802.11 bg without the ' $\mathrm{e}$ ' extension, two Ethernet switches and the PIX firewall in an open (i.e., not dedicated) network. The three depicted graphs correspond to three independent measurements performed in three short time intervals shifted by about 15 minutes one from another. Figure 2 presents the same RTT data as discrete-time functions. The considerable increase in RTTs during the first measurement (solid line) can be interpreted as the effect of an unidentified traffic in the shared network or disturbances. It is necessary to notice that the reduction of the sampling period can induce a significant increase in delays due to retransmissions, a contention procedure for media access control and buffering in network card drivers (see Fig. 8). These phenomena are exceptionally important for wireless media, but the problem exists in any shared media.

The problem can be diminished using multiple transmission channels and suitable traffic engineering mechanisms (Morawski, 2005), where link costs (delays) are considered uncertain numbers. From the above discussion it follows that the selection of the sampling period in network based control systems requires additional analysis compared with that performed (Åström and Wittenmark, 1997; Wang and Liu, 2008) in the case of classical computer controlled systems.

It is worth mentioning here that in our experiments we applied general-purpose inexpensive hardware typically used for office networking, and the ultimate goal of our research was to investigate if the application of such hardware enables successful deployment of NCSs with high dynamic controlled plants. In our experimental NCS as a plant there served an MLS, which is a structurally unstable system of high dynamics. From analytic deliberations and simulations of the stand-alone closed-loop control system with MLS it follows that stable operation of this system requires frequent sampling of the measured output and fast floating point computations of the control feedback. Hence, the compensation of the influence network introduced delays is critical for proper operation of an NCS with an MLS. Taking into account results concerning network introduced delays and requirements regarding the choice of the sampling period for digital control of the MLS, we have concluded that the statistical approach to the design of network based controllers for the MLS is less useful than the predictive control approach described by Liu et al. $(2005 ; 2007)$ and Yang et al. (2005).

\section{Other components of the link cost}

The quality of a transmission path (metric) is always a scalar function of attributes of all links that belong to the path. For every link, some number of attributes can be defined. Some of them have technical character, some othersnon-technical. To the last category belong policies and preferences which are only in the BGP protocol (Zhang and Bartell, 2004), and therefore they are not considered in our paper.

Although latency is the most obvious technical link quality measure (or attribute), especially for control applications, there exist others. Most popular, but practically unusable in our area of applications, is a bandwidth. The bandwidth (physical layer bit rate transmission) is significantly higher than a throughput (transport or application layer link speed), and the ratio depends on several factors like the media access procedure, the noise level, the number of stations competing for the channel access, etc., so it is too viable.

Another useful link metric vector components are the loss ratio including the drop ratio, that is a result of queues exhaustion, the real loss ratio that is a result of destruction of packets due to noise or interferences, the power necessary to transmit data, reliability, load, etc. (Willig et al., 2005). However, it is necessary to note that none of the link cost components is independent of others. These kinds of link attributes are especially useful when end or intermediate nodes are controlled by microcontrollers. 
In this case, the queueing of messages (main source of delays in traditional networks) is practically impossible due to resource limitations.

\section{Application layer compensation}

As mentioned in Section 1, we consider the NCS with the MLS which is an example of a control plant of high dynamics. It is well known (Khalil, 1996) that this control plant can be described by the non-linear state model, but our former results (Lewandowski, 2003; Morawski and Zajączkowski, 2005) show that, for the design of a state feedback controller responsible for the stabilisation of the MLS at the selected operating point, the linear state model obtained using Taylor's linearisation of the non-linear state and output equations is sufficiently accurate. Hence in the sequel we consider a discrete-time, linear, timeinvariant control plant modelled by the following state and output equations:

$$
\begin{aligned}
\mathbf{x}((k+1) \Delta) & =\mathbf{A} \mathbf{x}(k \Delta)+\mathbf{B u}(k \Delta), \\
\mathbf{y}(k \Delta) & =\mathbf{C} \mathbf{x}(k \Delta),
\end{aligned}
$$

where $\mathbf{x}(k \Delta) \in \mathrm{X}=\mathbb{R}^{n}$ is the state vector, $\mathbf{u}(k \Delta) \in$ $\mathrm{U}=\mathbb{R}^{m}$ is the vector of control inputs, $\mathbf{y}(k \Delta) \in \mathrm{Y}=\mathbb{R}^{p}$ is the vector of measured outputs, $\mathbf{A}, \mathbf{B}, \mathbf{C}$ are matrices of suitable dimensions and $\Delta$ is the sampling period.

Further we assume that the model of the plant is completely reachable and completely observable (Åström and Wittenmark, 1997). In other words, we assume that the pair $(\mathbf{A}, \mathbf{B})$ is completely reachable and the pair $(\mathbf{A}, \mathbf{C})$ is completely observable. From the first assumption it follows that using the pole shifting method or linearquadratic optimal control theory (Åström and Wittenmark, 1997), we can design a linear state feedback

$$
\mathbf{u}(k \Delta)=\mathbf{K}_{\mathrm{s}} \mathbf{x}(k \Delta), \quad \mathbf{K}_{\mathrm{s}} \in \mathbb{R}^{m \times n}
$$

such that the closed-loop system

$$
\mathbf{x}((k+1) \Delta)=\left(\mathbf{A}+\mathbf{B K}_{\mathbf{s}}\right) \mathbf{x}(k \Delta)
$$

is asymptotically stable with desired dynamic properties.

The second assumption guarantees that we can design a Luenberger observer (Åström and Wittenmark, 1997) which estimates the state vector of the plant on the basis of the measured output and control input. Further, we consider the implementation problem of the control law (2) using an NCS (Hristu-Varsekelis and Levine, 2005; Wang and Liu, 2008) shown in Fig. 3 .

A networked control system consists of a plant, controller and a computer network across which all sensor and actuator data must be sent (Hristu-Varsekelis and Levine, 2005). In this system, the plant is equipped with some computer responsible mainly for sending and receiving data to and from a remote controller using a communication channel provided by the computer network.

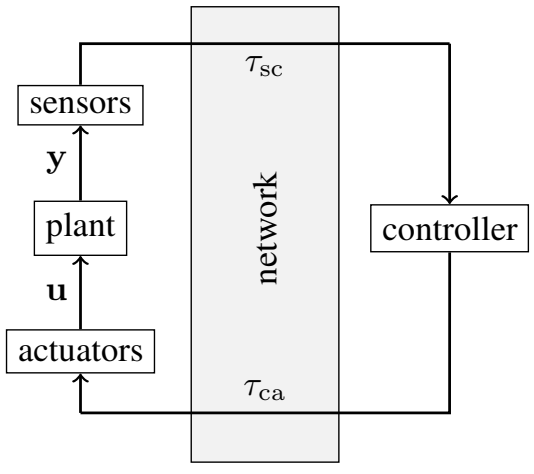

Fig. 3. One channel closed-loop NCS.

If a computer network is used to exchange information between the local computer and the remote controller, then some transmission delays are introduced into the feedback loop. We distinguish two transmission delays: the sensor to controller delay $\tau_{\mathrm{sc}}$ and the controller to actuator delay $\tau_{\text {ca }}$, and we assume that there exist nonnegative integers (Liu, Xia and Rees, 2005; Liu et al., 2007; Yang et al., 2005) $n_{\mathrm{sc}}$ and $n_{\mathrm{ca}}$ such that the total transmission delay $\tau_{\text {sa }}=\tau_{\text {sc }}+\tau_{\text {ca }}$ satisfies the inequality

$$
0<\tau_{\mathrm{sa}}<\Delta n_{\mathrm{sa}},
$$

where $n_{\mathrm{sa}}=n_{\mathrm{sc}}+n_{\mathrm{ca}}$

Remark 1. The delay $\tau_{\text {ca }}$ includes the time spent by the remote controller on the execution of the control algorithm.

The second assumption concerning the operation of the network is as follows: the number of lost packets on the route from the plant to the controller and on the route from the controller to the plant is less than $n_{\mathrm{sc}}$ and $n_{\mathrm{ca}}$, respectively. This assumption is required for the stability proof of the NCS (Liu et al., 2005; 2007)

The controller operates in the event driven mode. This means that a control algorithm is started when the new packet with measured data is received. Sensors work in the clock (time) driven mode, that is, samples of the measured output signals are computed at the time instants $t_{k}=k \Delta, k \in \mathbb{Z}, \mathbb{Z}$ being the set of integers. Actuators operate both in the clock and event driven modes. This means that control values are received when a packet from the controller arrives and the new control value is applied in the immediate sampling instant.

Let $\mathrm{T}_{k}=\left[t_{k}, t_{k+1}\right)$. In this time interval the local computer can receive zero, one or more (at most $n_{\mathrm{sa}}$ ) data packets with the new values of the control input. If during this interval no packet arrives, then the preceding value of the control input will be applied in the next time interval $\mathrm{T}_{k+1}=\left[t_{k+1}, t_{k+2}\right)$. If in the interval $\mathrm{T}_{k}$ the local computer receives more than one data packet with the control values, then the most recent value will be applied and earlier (i.e., based on the earlier sample, not received earlier) 
values will be neglected. These scenarios are illustrated in Fig. 4.

Suppose now that on the basis of the plant model (1) the linear state feedback (2) was designed. If this control law is used in the NCS in which random time-varying transmission delays occur, then control values are applied with the delay $r(k)$ which takes values from the set

$$
\mathcal{R}=\left\{1,2, \ldots, n_{\mathrm{sa}}\right\} .
$$

Note that $r(k) \in \mathcal{R}$ corresponds to the time instant $t_{k}=k \Delta$ and to the sample $\mathbf{y}(k \Delta)$ of the output. It is easy to see that the best situation occurs when $r(k)=1$, but even in this case the application of the new control value by the actuator is delayed. If in an NCS no mechanisms exist responsible for the compensation of the influence of the random delays introduced by the network, then the behaviour of the closed-loop system degrades significantly and in some cases such a system can suffer from stability loss. Hence, the compensation of the influence of this delay phenomenon belongs to the most important problems encountered in the area of network based control systems.

As mentioned above, the remote controller should be equipped with some mechanism compensating the influence of the transmission delays introduced by the computer network. One of the mechanisms proposed recently (Liu et al., 2005; 2007; Yang et al., 2005) is the method of prediction described shortly in what follows.

Consider the NCS depicted in Fig. 3 and assume that the local computer has a buffer for storing a finite number of control values. Assume also that the remote controller stores the model of the plant represented by the triple $(\mathbf{A}, \mathbf{B}, \mathbf{C})$.

Suppose that the controller received the sample $\mathbf{x}(k \Delta)$ of the plant state together with the time stamp $k$ corresponding to this sample.

If we know the state $\mathbf{x}(k \Delta)$, then using (2) we can compute the control value $\mathbf{u}(k \Delta)$. The next predicted value $\hat{\mathbf{x}}((k+1) \Delta)$ of the state can be computed according to the equation

$$
\hat{\mathbf{x}}((k+1) \Delta)=\mathbf{A} \mathbf{x}(k \Delta)+\mathbf{B u}(k \Delta) .
$$

Applying (2) again we obtain the predicted value $\mathbf{u}((k+1) \Delta)$ of the control signal

$$
\mathbf{u}((k+1) \Delta)=\mathbf{K}_{\mathrm{s}} \hat{\mathbf{x}}((k+1) \Delta) .
$$

Repeating these computations $n_{\mathrm{sa}}-1$ more times with a respective exchange of arguments gives the last predicted values of the state and control

$$
\begin{aligned}
\hat{\mathbf{x}}\left(\left(k+n_{\mathrm{sa}}\right) \Delta\right)= & \mathbf{A} \hat{\mathbf{x}}\left(\left(k+n_{\mathrm{sa}}-1\right) \Delta\right) \\
& +\mathbf{B u}\left(\left(k+n_{\mathrm{sa}}-1\right) \Delta\right), \\
\mathbf{u}\left(\left(k+n_{\mathrm{sa}}\right) \Delta\right)= & \mathbf{K}_{\mathrm{s}} \hat{\mathbf{x}}\left(\left(k+n_{\mathrm{sa}}\right) \Delta\right) .
\end{aligned}
$$

As a result, we obtain the finite sequence of control values $\mathbf{u}(k \Delta), \mathbf{u}((k+1) \Delta), \ldots, \mathbf{u}\left(\left(k+n_{\mathrm{sa}}\right) \Delta\right)$.

This sequence with the first element omitted and the corresponding time stamp $k$ attached is sent via the computer network to the local computer. This computer compares its current discrete time with the received time stamp and computes the value of $r(k)$. From the control sequence, the element of the index $r(k)$ is selected and applied in the immediate sampling instant (Fig. 4).

It is easy to notice that the linear control (2) is based on the assumption that the entire state vector is available for measurement. In many control plants encountered in practice, one can measure only the output of the plant, as is the case with our laboratory MLS, where only the ball position (the height of levitation) is measured (Morawski and Zajączkowski, 2005; 2007a; Lewandowski, 2003). In such a situation the control law (2) cannot be realised directly and should be supplemented by some state estimation mechanism. In the case of deterministic linear systems, the well-known and efficient method of state estimation is the application of the full-order Luenberger observer (Åström and Wittenmark, 1997) described by the discrete-time state equation of the form

$$
\begin{aligned}
\mathbf{x}_{\mathrm{o}}((k+1) \Delta)= & \mathbf{A x}_{\mathrm{o}}(k \Delta)+\mathbf{B u}(k \Delta) \\
& +\mathbf{K}_{\mathrm{o}}(\mathbf{y}(k \Delta)-\mathbf{C x}(k \Delta)),
\end{aligned}
$$

where $\mathbf{x}_{\mathrm{O}} \in \mathbb{R}^{n}$ is the state of the observer and $\mathbf{K}_{\mathrm{o}} \in$ $\mathbb{R}^{n \times p}$ is the gain matrix.

This type of observer was used by Liu et al. (2005; 2007 ) in their discussion concerning predictive approach for the design of stable network based control systems and was implemented in our remote controller of MLS.

The experimental MLS described by Lewandowski (2003) as well as Morawski and Zajączkowski (2007a; 2007b) consists of an iron ball suspended under an electromagnet supplied by the voltage controlled power source.

It is well known (Khalil, 1996) that the dynamics of this system can be described by a non-linear state equation in which the ball position, ball velocity and the current of electromagnet winding are the state coordinates, and the output voltage of the power source is the control input. In our system the ball position is the only measured quantity, so our system belongs to the class of non-linear single input single output (SISO) systems with control appearing linearly (Morawski and Zajączkowski, 2005). Taylor's linearisation of this system at a selected operating point gives a linear time-invariant SISO continuous-time system with one positive real eigenvalue. To be more specific, the eigenvalues of our laboratory MLS are 74, -58.5, -145 . Hence this system is unstable and of high dynamics.

The experimental MLS is connected to a typical PC (local computer) that reads data from the height sensor and controls the voltage applied to the winding of the electromagnet. The height of levitation is measured by the optical 


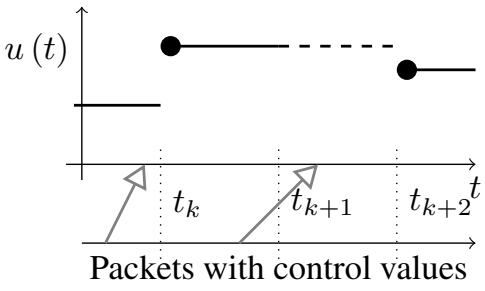

(a)

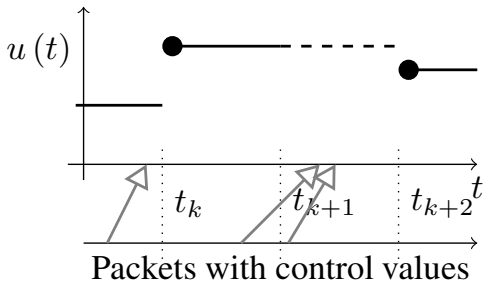

(b)

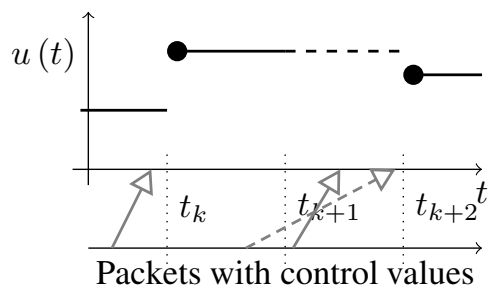

(c)

Fig. 4. Possible scenarios of the packet arrival in the interval $\mathrm{T}_{k}$ : no packet with control data is received in the specified interval (a), two packets with control data are received in the same interval (b), an order of the packet arrival is irrelevant (c).

sensor connected to a 12-bit AD converter. The local computer sends to and receives data from the remote computer system responsible for the computation of the control values. These two computers exchange data via a computer network using UDP packets. The local computer sends packets at the rate of 1024 packets per second. Each packet contains the sample of the height of levitation, the corresponding time stamp and the respective control value, so the size of this packet is $40 \mathrm{~B}$, which gives the required throughput of $688128 \mathrm{bps}$ for the Ethernet network.

The packets produced by the remote controller are asynchronously sent to the local computer and can be considered responses to the packets containing samples of the plant output. Each packet sent by the remote controller contains a copy of the respective time stamp and the finite sequence of 11 control values computed according to the method of prediction as described earlier. From this it follows that the size of the UDP packet is $72 \mathrm{~B}$, which results in the throughput estimate of 901120 bps. The estimates of throughput are sufficient to perform data transmission using typical modern Ethernet or WiFi networks, though in the case of WiFi the communication is half duplex and additional time for a media access procedure is necessary. The local computer evaluates the difference between its current time and the received time stamp. This quantity interpreted as the round trip time is expressed in milliseconds and used as an index needed for the selection of the suitable element from the received sequence of control values.

Figure 6 presents the graphs of the ball position when the network based control loop uses the Ethernet and WiFi networks, respectively. The results of the operation of network based systems are compared with that of a standalone system. It is worth explaining here that the control algorithm performed by the standalone system does not contain the full-order observer and that the state vector is estimated using discretised linear state equations of the plant. As can be seen in Fig. 6, this method of state estimation is of rather poor quality and in network based systems was replaced by a full-order Luenberger observer possessing well-known filtering capabilities 4 .

\footnotetext{
${ }^{4}$ The implementation of the observer on a local computer is impos-
}

Figure 5 shows the subsequent delays seen by the actuator.

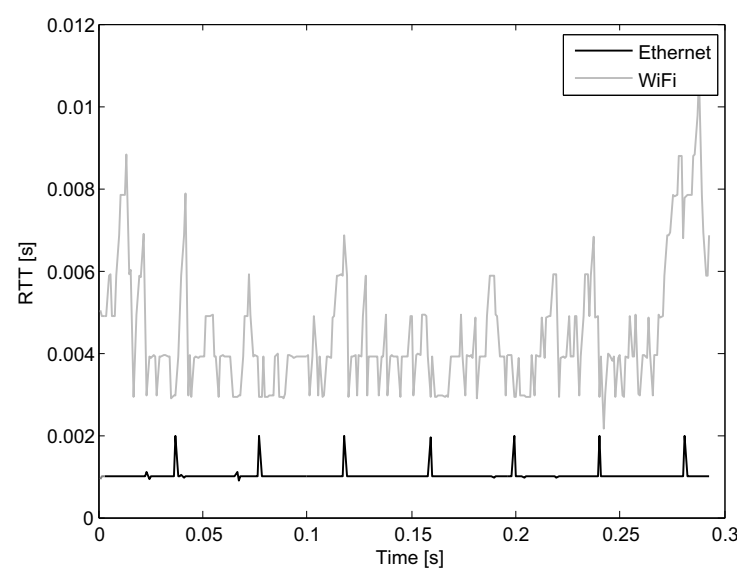

Fig. 5. RTT seen by the actuator.

\section{Network layer compensation}

The described method of network induced delays compensation has limited capabilities because, while increasing $n_{\text {sa }}$, the quality of prediction and plant state estimation performed by the observer degrades significantly. Although theoretically $n_{\text {sa }} \in \mathbb{N}$ can take any value, in practice this number must be limited due to the inaccuracy of plant modelling, and in the case of our MLS it should take values in the interval $[1,15]$. Usually, occasional violation of this limit does not result in system misbehaviour, but in the case of bursty delays of losses of data series a system failure is practically unavoidable. Therefore, network layer compensation should be applied as a complementary mechanism. In our paper we neglect the important problem of proper queueing in terminal and intermediate nodes, although the effect of this phenomenon can be extremely dangerous (see Fig. 8). We refer the interested reader to the appropriate literature (e.g., Grzech, 2002). Here we shortly describe another method (Moraw-

sible due to resource limitations, especially the lack of a floating point unit. 


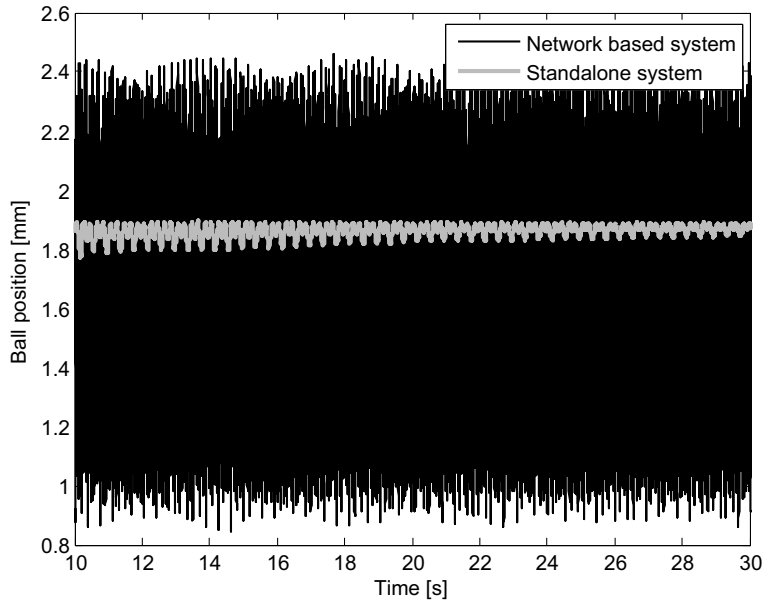

(a)

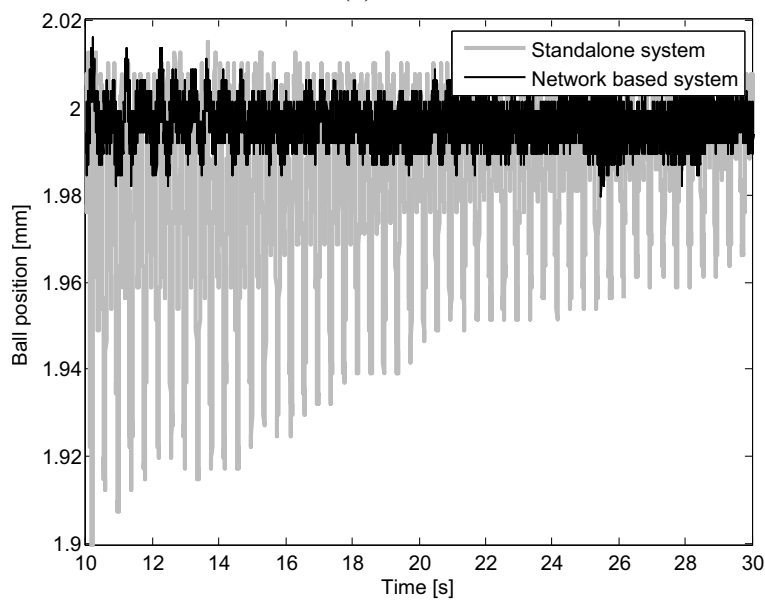

(c)

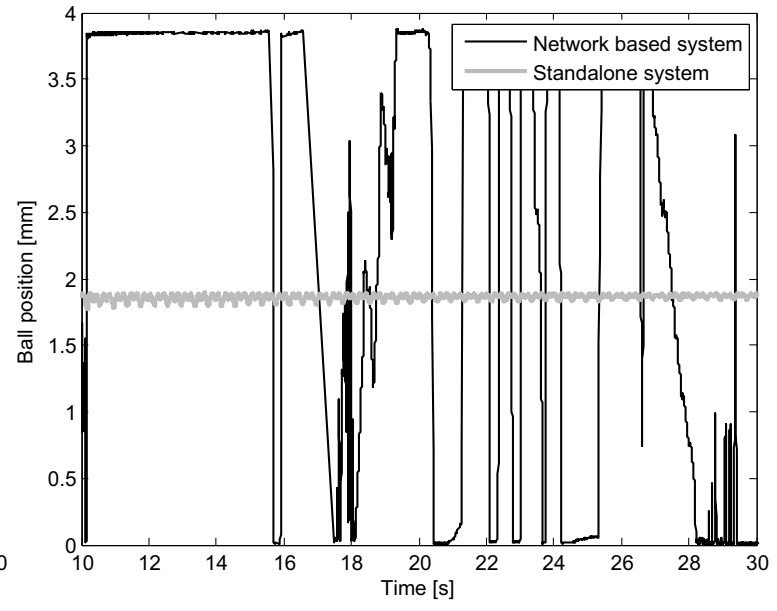

(b)

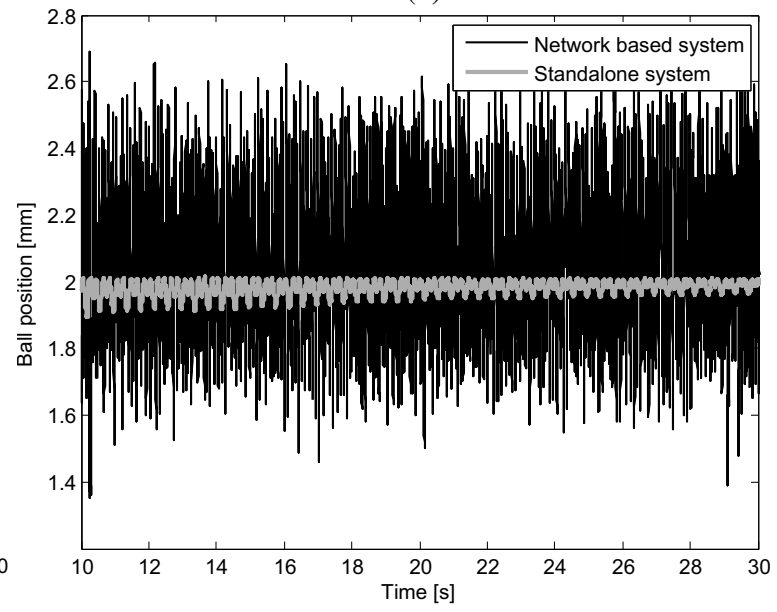

(d)

Fig. 6. Effect of application layer compensation in the MLS. Top panels: without compensation, bottom panels: with compensation. Left panels: Ethernet only based network, right panels: mixed Ethernet/802.11 network. Better operation of the NCS in (c) is the result of using the full-order observer for state estimation in Ethernet based systems. A WiFi based controller without compensation does not work at all (b).

ski, 2005; 2006b; 2007a; 2007b). of the utilisation of multiple channels, which alleviates both queueing and media access lags.

As mentioned in Sections 2 and 3 , the quality of links can be expressed as overall latencies of transmission by particulars links. These latencies are highly variable quantities and constitute the sum of propagation, transmission, media access, processing and queueing times. The propagation, transmission and processing times are less or more constant, while the remaining ones are not. Therefore, we use the queue size only (and the remaining constant times) as an input variable $\eta(s)$ of a simple first order filter:

$$
\xi(s+1)=\alpha \eta(s)+(1-\alpha) \xi(s)
$$

where $s$ is an integer that describes the subsequent time instants and $\xi(s)$ is a "smoothed" $\eta(s)$. The value of $\eta(s)$ can be considered a temporary cost of the link 5 . This cost can be defined arbitrarily, but taking into account Gallager's principle (Gallager, 1977) we decided to define $\eta(s)=d+q(s) / t r$, where $q(s)$ is the link queue length at time instant $s, t r$ is the depletion ratio, and $d$ is constant (propagation and processing times). Note that the media access time influences $q(s)$ exactly in the same way as enqueuing packets (if no packets are dropped). Therefore, $q(s)$ can be considered a congestion indicator. This approach is similar to the one applied in TCP FAST (Wei et al., 2006). Additionally, we take into consideration the

\footnotetext{
${ }^{5}$ Note that the cost of a link is typically some constant, but in the solution presented here it is highly varying (Fig.9.
} 
dynamics of $\xi(s)$ :

$$
\begin{array}{r}
v^{+}(s+1)=\beta(\eta(s)-\xi(s))+(1-\beta) v^{+}(s) \\
\text { if } \eta(s) \geq \xi(s), \\
v^{-}(s+1)=\beta(\xi(s)-\eta(s))+(1-\beta) v^{-}(s) \\
\text { otherwise. }
\end{array}
$$

For statistically invariant systems, we have $v^{+}(s)=$ $v^{-}(s)$ (definition of variance), but our network certainly does not fulfil this equality (Welzl, 2005). The result of the above approximation is presented in Fig. 9 . The values of $\xi(s), v^{+}(s), v^{-}(s)$ create an uncertain value that can describe the link quality (or the link cost), where $\xi(s)$ is the central (most likely) value, and $v^{+}(s), v^{-}(s)$ are bounds of uncertainties above and below $\xi(s)$, respectively. The sum of the costs along the path (uncertain as well) defines its metric.

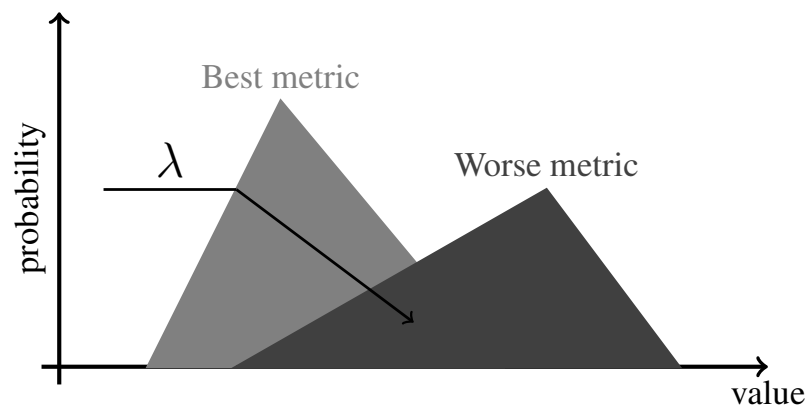

Fig. 7. Geometrical interpretation of the probability of forwarding packets using worse metrics.

Two different uncertain values can have the common part (Fig. 7) $-\lambda$. The packets are forwarded randomly, inversely proportional to the values of $\lambda$, defined for every path not including loops. Equations (10) and (11) are stable for any values $\alpha, \beta \in(0,1)$, and theoretically the best behaviour can be obtained when $\alpha=\beta$. However, many experiments with these algorithms show that for $\beta=2 \alpha$ better performance is obtained, exactly as in the TCP protocol (Stallings, 2002). The value of $\alpha$ should follow the variability of the link load and needs to be adjusted. This adjustment is performed when the current $\xi(s)$ is outside bounds $\left(\xi(p)-v^{-}(p), \xi(p)+v^{+}(p)\right)$, where $p$ is the time instant of the last change, and thus $\alpha$ and $\beta$ are piecewise constant. In the papers by Morawski $(2005 ; 2006 b ; 2007 a ; 2007 b)$ the methods of adaptive tuning of the proper values of $\alpha$ and $\beta$ are described. Our strategy significantly decreases the probability of burst delay or losses and the susceptibility on congestion, noise, interferences, etc.

Our approach allows incorporating an additional degree of system resiliency: standby. Taking the advantage of multicast communication, there is a possibility to use multiple remote computers simultaneously. After a link failure (or simply after changing the link cost), the faster

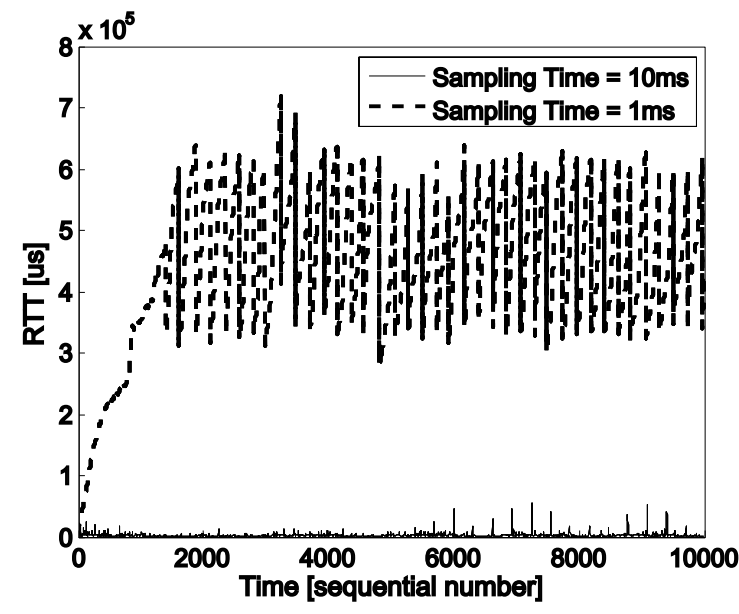

Fig. 8. Effect of the overloading of some (wireless) link. Very long inescapable delays cause system failure.

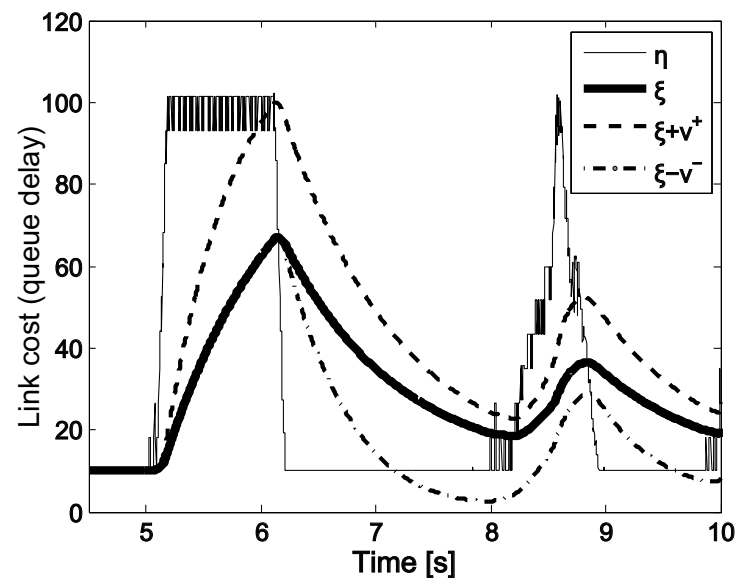

Fig. 9. Effect of link cost approximation of some link. The peak on the right is the effect of a dissipation of a congestion (simulation using an NS2 simulator).

answer from the remote computer is taken into account without significant problems with system stability.

The scheme of the redundant active standby system and the process of switching an active controller of the MLS is presented in Fig. 10

\section{Conclusions}

A new approach for robustness enhancement of NCSs against closed-loop latencies has been presented. In our opinion, the novelty of this approach is based on a proper combination of the predictive control method of controller design and recently developed methods of traffic engineering. The efficiency of the proposed approach was verified by a number of experiments performed using our laboratory NCS based on Ethernet and WiFi networks and implemented using standard inexpensive network hardware typically applied for office networking. It is worth em- 


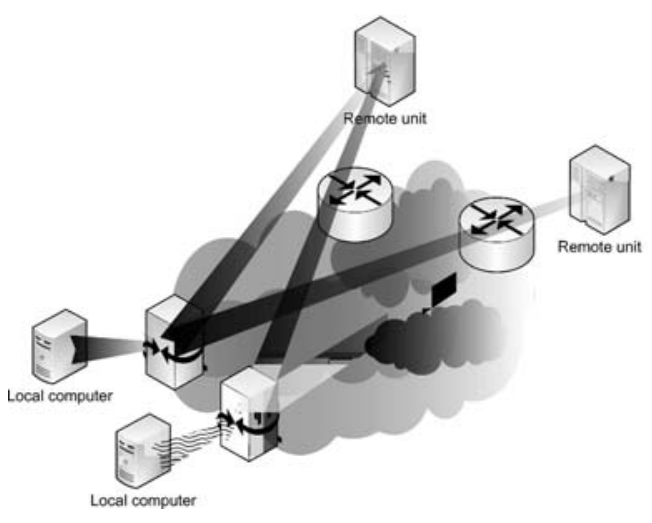

(a)

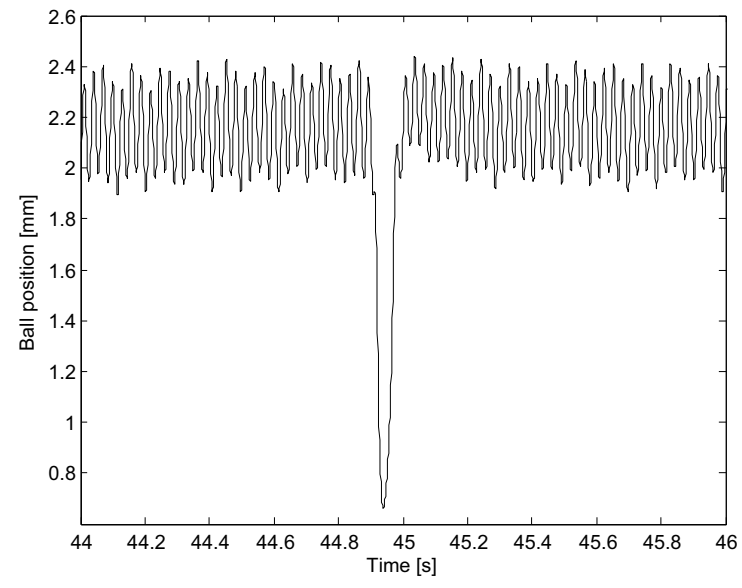

(b)

Fig. 10. Application layer redundancy: scheme of the system (a), process of switching the active remote controller (b).

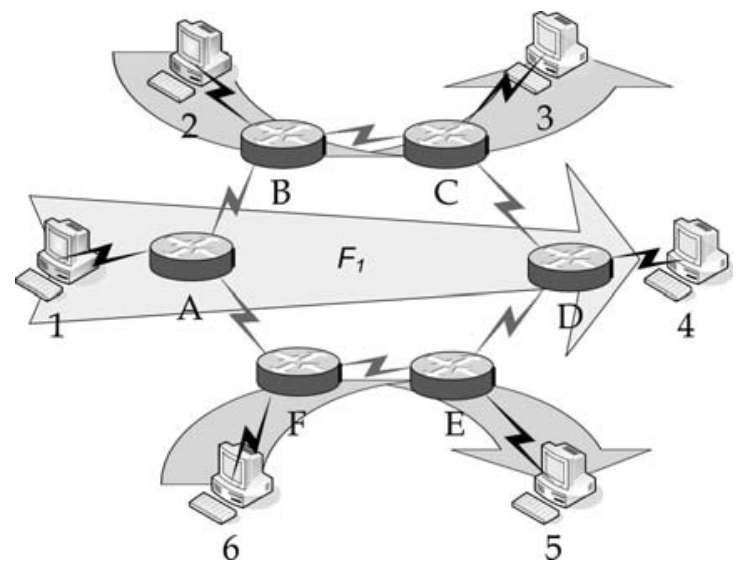

Fig. 11. Test topology for the network layer test algorithm. Inner links have 0.1 of the capacity of radial links.

phasising that in our NCS as a control plant served the magnetic levitation system, which is an example of a structurally unstable system of high dynamics, and that these properties of the control plant made the design of the robust NCS additionally demanding. Experimental results have confirmed our hypothesis that the robustness of an NCS against network induced latencies cannot be guaranteed by the design of a controller alone and should be supplemented by properly selected methods of traffic engineering.

Although similar results can be obtained using dedicated real-time networks like Profibus, real-time versions of Ethernet, and many others, the proposed combination of methods is in fact independent of link layer technologies and works correctly on top of existing and future link layer implementations including wireless ones. Therefore, such an implementation is very cost effective because it is realised only by software.

One of possible directions of further research is the application of the proposed traffic engineering algorithms within dynamic environments, e.g., a set of vehicles.

\section{References}

Åström, K. and Wittenmark, B. (1997). Computer-Controlled Systems, 3rd Edn., Prentice Hall, Upper Saddle River, NJ.

ARINC (1990). ARINC Specification 629, Multi-transmitter data bus, Aeronautical Radio INC, Annapolis, MD.

Decotignie, J.-D. (2005). Ethernet-based real-time and industrial communications, Proceedings of IEEE 93(6): 1102-1117.

Gallager, R. (1977). A minimum delay routing algorithm using distributed computation, IEEE Transactions on Communication 25(1): 73-84.

Grzech, A. (2002). Traffic Control of Teleinformatic Networks, Wrocław University of Technology Press, (in Polish).

Hristu-Varsekelis, D. and Levine, W.S. (2005). Handbook of Networked and Embedded Control Systems, Birkhäuser, Boston, MA.

http: / /www.worldfip.org (n.d.). Factory Instrumentation Protocol.

Khalil, H.K. (1996). Nonlinear Systems, 2nd Edn., Prentice Hall, Upper Saddle River, NJ.

Khanna, A. and Zinky, J. (1989). The revised ARPANET routing metric, Proceedings of the SIGCOMM Symposium on Communications Architectures \& Protocols, Austin, TX, USA, pp. 45-56.

Lewandowski, D. (2003). Magnetic levitation system, Master's thesis, Technical University of Łódź, Division of Computer Networks, (in Polish), http: / /www.zsk.p.lodz.pl /morawski/Dyplomy/Lewandowski.pdf

Liu, G.P., Xia, Y., Chen, J., Rees, D. and Hu, W. (2007). Networked predictive control of systems with random network delays in both forward and feedback channels, IEEE Transactions on Industrial Electronics 54(3): 1282-1297. 
Liu, G. P., Xia, Y. and Rees, D. (2005). Predictive control of networked systems with random delays, Proceedings of the 16th IFAC World Congress, Prague, Czech Republic, (no. We-M15_TO/2).

Liu, W., Lou, W. and Fang, Y. (2005). An efficient quality of service routing algorithm for delay-sensitive applications, Computer Newtworks 47(1): 87-104.

Mitchell, R. (2004). Profibus: A Pocket Guide, International Society of Automation, Research Triangle Park, NC.

Montestruque, L. and Antsaklis, P. (2003). On the model-based control of networked systems, Automatica 39(10): 18371843.

Montestruque, L. and Antsaklis, P. (2005). Handbook of Networked and Embedded Control Systems, Birkhäuser, Boston, MA, pp. 601-625.

Morawski, M. (2005). Uncertain metrics applied to QoS multipath routing, Proceedings of the 5th International Workshop on Design of Reliable Communication Networks, DRCN'05, Island of Ischia, Naples, Italy, pp. 353-360.

Morawski, M. (2006a). Analysis of short latencies in industrial network environments, Journal of Applied Computer Sciences 14(2): 65-78.

Morawski, M. (2006b). Optimal adaptive routing with efficient flapping prevention, 4th Polish-German Teletraffic Symposium, PGTS, Wroctaw, Poland, pp. 85-94.

Morawski, M. (2007a). Traffic engineering for industrial networks, Proceedings of the 14th Polish Teletraffic Symposium, Zakopane, Poland, pp. 151-164.

Morawski, M. (2007b). Traffic engineering for industrial networks, Theoretical and Applied Informatics 19(4): 239-254.

Morawski, M. and Zajączkowski, A.M. (2005). Control of the magnetic levitation system using linear and non-linear output feedback, Proceedings of the 7th Conference on Control in Power Electronics and Electrical Drives, SENE, Łódź, Poland, pp. 367-372.

Morawski, M. and Zajączkowski, A.M. (2007a). Predictive control of the magnetic levitation system using a network based controller. Part 1: Control algorithm, Proceedings of the 8th Conference on Control in Power Electronics and Electrical Drives, SENE, Łódź, Poland, pp. 335-340.

Morawski, M. and Zajączkowski, A.M. (2007b). Predictive control of the magnetic levitation system using a network based controller. Part 2: Experimental results, Proceedings of the 8th Conference on Control in Power Electronics and Electrical Drives, SENE, Łódź, Poland, pp. 341-346.

Nutzerorganisation, P. (2006). Profinet technology and application, Technical report, PROFIBUS \& PROFINET International (PI), Karlsruhe.

Pfeifer, O., Ayre, A. and Keydel, C. (2003). Embedded Networking with CAN and CANopen, RTC Books, Renton, WA.
Stallings, W. (2002). High-Speed Networks and Internets: Performance and Quality of Service, 2nd Edn., Prentice Hall, Upper Saddle River, NJ.

Wang, F. Y. and Liu, D. (2008). Networked Control Systems. Theory and Applications, Springer, New York, NY

Wei, D. X., Jin, C., Low, S. H. and Hegde, S. (2006). FAST TCP: Motivation, architecture, algorithms, performance, IEEE/ACM Transactions on Networking 14(6): 12461259.

Welzl, M. (2005). Network Congestion Control. Managing Internet Traffic, Wiley \& Sons, Hoboken, NJ.

Willig, A., Matheus, K. and Wolisz, A. (2005). Wireless technology in industrial networks, Proceedings of IEEE 93(6): 1130-1151.

Yang, Y., Wang, Y. and Yang, S.-H. (2005). A networked control system with stochastically varying transmission delay and uncertain process parameters, Proceedings of the 16th IFAC World Congress, Prague, Czech Republic, (no. WeM15_TO/3).

Zhang, R. and Bartell, M. (2004). BGP Design and Implementation, Cisco Press, Indianapolis, IN.

Zurawski, R. (2009). Networked Embedded Systems, 2nd Edn Industrial Information Technology, CRC Press, New York, NY.

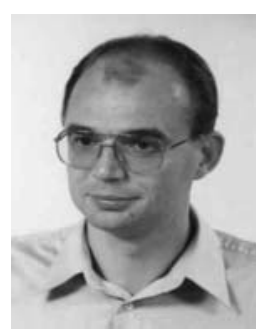

Michat Morawski received the M.Sc. degree from the Technical University of Łódź, Poland, in 1990, and the Ph.D. degree in 1997 from the Institute of Electrical Engineering in Warsaw, Poland. He is currently with the Technical University of Łódź (Institute of Information Technology). His research activity includes traffic engineering in computer networks, industrial information systems and their practical implementation.

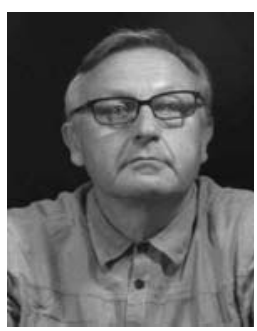

Antoni M. Zajączkowski received the M.Sc. and $\mathrm{Ph} . \mathrm{D}$. degrees in electrical engineering in 1973 and 1979, respectively, both from the Technical University of Łódź, Poland. From 1978 to 2003 he served as an assistant professor at the Division of Fundamental Research in Electrical Engineering of the Polish Academy of Sciences. In 1997 he joined the Technical University of Łódź, where he currently serves as the deputy director of the Institute of Information Technology. Dr. Zajączkowski has authored and coauthored many research papers on applications of nonlinear control theory in power systems and AC electrical drives, and on networked based control systems.

Received: 2 December 2009 Revised: 5 July 2010 